\title{
Narrativa
}

\section{Nuove frontiere del Sud. Genesi e sviluppo di un pensiero plurale sul Sud nella letteratura e nella cultura dell'Italia contemporanea}

Margherita Marras e Giuliana Pias

\section{CpenEdition}

\section{Journals}

Edizione digitale

URL: https://journals.openedition.org/narrativa/614

DOI: $10.4000 /$ narrativa.614

ISSN: 2804-1224

Editore

Presses universitaires de Paris Nanterre

\section{Edizione cartacea}

Data di pubblicazione: 1 décembre 2017

Paginazione: 7-12

ISBN: 978-2-84016-289-6

ISSN: 1166-3243

Notizia bibliografica digitale

Margherita Marras e Giuliana Pias, «Nuove frontiere del Sud. Genesi e sviluppo di un pensiero plurale sul Sud nella letteratura e nella cultura dell'Italia contemporanea», Narrativa [Online], 39 | 2017, online dal 01 décembre 2021, consultato il 14 janvier 2022. URL: http://journals.openedition.org/narrativa/ 614 ; DOI: https://doi.org/10.4000/narrativa.614

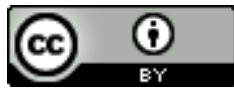

Narrativa est mise à disposition selon les termes de la Licence Creative Commons Attribution 4.0 International. 


\section{Nuove frontiere del Sud. \\ Genesi e sviluppo di un pensiero plurale sul Sud \\ nella letteratura e nella cultura dell'Italia contemporanea}

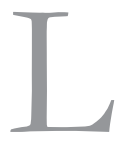

'ormai sentiero battuto del Sud considerato "molto più che un semplice punto cardinale" o ricondotto a una pura costruzione convenzionale acquista uno specifico significato a seconda dello spazio che viene definito o del luogo da cui tali formulazioni concettuali provengono. Fatto inconfutabile è che ogni luogo geografico ha un "suo" Sud, la cui localizzazione è strettamente correlata al punto d'osservazione che gli è proprio (seppure si possano reperire delle premesse e delle caratteristiche definitorie comuni a diversi territori). Nel caso dell'Italia, la costruzione e la percezione del "suo" Sud è stata storicamente vincolata a prospettive geo-economiche, geo-sociali e geo-culturali inscindibili dalla costante contrapposizione al "suo" Nord, divenuto un binomio antitetico e un elemento sintomatico della condizione di subalternità del Meridione, fulcro e fonte imprescindibile di pensieri politici, economici, sociologici e filosofici, nonché chiave di lettura privilegiata dei processi culturali, identitari, artistici e letterari.

Per questo motivo, risulta impossibile affrontare la questione del Sud come soggetto in ambito italiano facendo astrazione da una serie di premesse concatenate e reciprocamente coestensive. La prima di queste ci porta a individuare la collocazione geografica altamente strategica dell'Italia, e in particolare quella del "suo" Sud, posizionata al centro di quel bacino Mediterraneo che adempie al tradizionale status di canale di comunicazione tra diversi continenti, aprendosi all'irriducibile complessità di mare-frontiera (muro) e mare-cerniera (ponte).

Assurto a laboratorio ideologico negli anni' 90 , il Mediterraneo ha rappresentato per numerosi studiosi uno dei luoghi d'elezione del post-coloniale, "il luogo della misura, dove era possibile decostruire le tentazioni di ogni fondamentalismo, riprendendo il filo delle mille contaminazioni e dei mille ibridi nati sulle 
sue sponde". A emergere con insistenza, in questi anni, è la prospettiva di un Mediterraneo non più legato all'arretratezza, all'abbandono e al degrado, ma piuttosto a una visione del mondo alternativa,

capace di battere tutti i fondamentalismi, compreso quello della crescita ininterrotta e dell'individualismo competitivo che costituiva il nucleo costitutivo dell'identità dei vincitori. L’idea era di avviare la costruzione di una koinè culturale/politica, una convivenza paritaria tra le culture che provasse ad associare i popoli affacciati su quel mare ${ }^{2}$.

Questo stesso richiamo alla ri-formulazione dei rapporti tra l'Europa e la sponda Sud del Mare Nostrum è all'origine di una serie di progetti della politica nostrana beceramente naufragati, come testimonia l'azione purtroppo limitata e politicamente inconsistente dell'Unione per il Mediterraneo, che avrebbe dovuto sottendere la virtuosa capacità di far dialogare tra loro tanto le diversità etniche quanto i saperi umanistici e politecnici sviluppatisi nella culla del Mediterraneo.

Non è quindi un caso che l'apertura di questo volume sia affidata a Franco Cassano che offre un'interessantissima revisione del presupposto epistemologico del "pensiero meridiano" da lui teorizzato nel suo celebre saggio dall'omonimo titolo. ${ }^{3}$ Pur mantenendo inalterato il significato ideale di solidarietà e speranza della sua prima proposta, Cassano ne rivede ora la portata alla luce degli avvenimenti e delle complicazioni storiche intercorse dalla prima pubblicazione del suo libro ad oggi, un arco di tempo che "si è dimostrato più avaro, premiando [...] molto più i costruttori di muri che quelli di ponti" ${ }^{4}$.

Nuove frontiere del Sud propone un numero importante di saggi che discernono l'oggettivazione del Sud nella sua dimensione frattale, frutto di una prospettiva tanto internazionale quanto endogena. Sebbene in molte delle analisi proposte s'incrocino periodi e sguardi diversi (fra Nord, Sud, Est e Ovest), il filo rosso che le accomuna è percettibile nella costante preoccupazione cassaniana di restituire al Sud l'antica dignità di soggetto del pensiero, che va di pari passo con la rimessa in discussione della parte oscura e imperfetta di quell'Occidente egemonico, ricettacolo di fondamentalismi culturali economici e etnocentrici, di cui anche le sue periferie hanno patito.

1. Cassano, Franco, "En Attendant Méditerranée", nel presente volume, pp. 13-27.

2. Ibid., p. 15.

3. ID., Il pensiero meridiano, Bari, Laterza, 1996.

4. ID., "En Attendant Méditerranée”, cit., p. 15. 
La scommessa iniziale e l'ambizione di questo volume nascono dall'esigenza di proporre alcune interpretazioni teoriche, letterarie, figurative, politiche e storiografiche sulle nuove frontiere del Sud inteso sia come oggetto sia come soggetto, ovvero in quanto produttore di pensieri plurali sul Sud e del Sud. Questi presupposti iniziali si sono tradotti in analisi accurate in cui tale pluralità viene evidenziata da delle riflessioni empiriche e teoriche connesse al Sud italiano, oggi obliquamente universalizzabili a un Sud globale.

Si moltiplicano così nel volume i riferimenti a Antonio Gramsci e alla questione meridionale, divenuta un esplicito modello su scala planetaria a opera della ricca corrente di pensiero dei Postcolonial Studies che, poco sensibile alla valenza propriamente geografica attribuita al Sud, ne focalizza il distinguo attorno alla posizione di spazio, reale e simbolico, generato dal confine e dal conflitto tra egemonico ed egemonizzato ${ }^{5}$, o detto altrimenti, dalla visione ideologica diffusa da un gruppo egemone che esercita la propria supremazia culturale, morale e politica sulle masse subalterne ${ }^{6}$. La ripresa del pensiero gramsciano si fa quindi attorno al concetto della subalternità, che diventa sostrato tanto della rivisitazione geografico-territoriale delle coordinate tradizionali di Nord e di Sud quanto della lettura delle nuove conformazioni del mondo-Sud negli autori post-coloniali. Così è per Edward Said:

Un esplicito modello geografico ci è offerto dal saggio di Gramsci Alcuni temi della quistione meridionale. Assai poco letto e studiato, è l'unico studio di analisi politica e culturale scritto da Gramsci (sebbene non lo abbia mai terminato); l'autore cerca di dare una risposta ai suoi compagni che gli hanno posto l'interrogativo, drammatico sia per l'azione che per il pensiero, su come pensare,

5. Gramsci, Antonio, Quaderni del carcere, in Gerratana, Valentino (a cura di), Torino, Einaudi, 1975, pp. 1419-1420.

6. Si tratta di un Sud derivante dalla visione ideologica che sta alla base dell'antropologia positivista e di una vasta produzione letteraria "meridionalista" che fonda e diffonde il discorso razzista su basi scientifiche. Un Sud che nutre anche un linguaggio che riflette gli stereotipi culturali (del "selvaggio", dell"“esotico", ecc.) e legittima l'“alterità" del Sud. Gramsci scrive: "E noto quale ideologia sia stata diffusa in forma capillare dai propagandisti della borghesia nelle masse del Settentrione: il Mezzogiorno è la palla di piombo che impedisce più rapidi progressi allo sviluppo civile dell'Italia; i meridionali sono biologicamente degli esseri inferiori, dei semibarbari o dei barbari completi, per destino naturale; e se il Mezzogiorno è arretrato, la colpa non è del sistema capitalistico o di qualsivoglia altra causa storica, ma della natura che ha fatto i meridionali poltroni, incapaci, criminali, barbari, temperando questa sorte matrigna con la esplosione puramente individuale di grandi geni, che sono come le solitarie palme in un arido e sterile deserto" (Gramsci, Antonio, Alcuni temi sulla questione meridionale, in ID. La questione meridionale, nuova antologia a cura e con introduzione di Nando dalla Chiesa, Milano, Melampo, 2014, p. 112). 
intendere e studiare l'Italia meridionale, visto che la sua disgregazione sociale la faceva apparire incomprensibile eppure paradossalmente fondamentale per una comprensione del nord. La brillante analisi di Gramsci va oltre il significato tattico che aveva tale questione per la politica italiana del 1926, dal momento che costituisce il culmine della sua attività giornalistica fino a quel momento e d'altra parte prelude ai Quaderni nei quali, a differenza del suo grande contemporaneo Lukács, egli mette a fuoco le fondamenta territoriali, spaziali e geografiche della vita sociale?

La modernità dei pensatori italiani e l'estensibilità dei loro pensieri sul Sud a categoria analitica del Sud globale sono altresì evidenti nel "tarantismo" di Ernesto de Martino e nelle influenze sartriana, heideggeriana e fanoniana che hanno nutrito la "crisi della coscienza" della sua scrittura, attualizzando la trasversalità e la post-colonialità del suo pensiero. Un altro riferimento imprescindibile è Pier Paolo Pasolini con le sue contestazioni rivolte alla tradizionale polarizzazione Nord-Sud e con la sua opposizione contro lo sguardo portato dall'Occidente industrializzato ed egemonico sul Sud povero e subalterno, la cui attualità e interesse permangono, nonostante i numerosi sconvolgimenti legati alla globalizzazione.

E fatto oramai acquisito che l'avvento di grandi potenze non occidentali abbia rimesso in discussione la ricorrente e tradizionale ripartizione bipolare o tripolare del mondo così come l'emergere di correnti di pensiero eterogenee che, legate agli sviluppi dell'antropologia culturale o a fattori di natura ideologica, hanno sollecitato una ridefinizione del Sud. Prova ne siano le importanti variazioni attinenti al tradizionale schema bipolare Nord-Sud, la cui causa si può attribuire al rimescolamento globalizzante degli ordini tradizionali, che si tratti di quelli ereditati culturalmente dall'epoca coloniale o di quelli costituitisi nella seconda metà del Novecento in seguito alla creazione dei due blocchi EstOvest. Non a caso, uno degli effetti perversi della globalizzazione è strettamente correlato alle sue "omogeneizzazioni imperfette", evidenti soprattutto nelle sempre più presenti diseguaglianze socio-economiche e di dominio che, se un tempo erano cristallizzate attorno al Sud e al Nord, ora sono leggibili a livello planetario. In altri termini, se l'universo simbolico classicamente collegato al Sud è poco evoluto (povertà, subalternità, ineguaglianza, dominazione subita), esso non è più sovrapponibile alle classiche frontiere che delimitavano questa realtà geografica.

7. SAID, Edward W., Cultura e imperialismo. Letteratura e consenso nel progetto coloniale dell'Occidente, Roma, Gamberetti, 1998 [1993], p. 74. 
Le tradizionali demarcazioni bipolarizzate del mondo scaturite da rapporti egemonici (centro e periferia, mondo dominante e mondo dominato, ecc.) sono state quindi scalzate dai processi legati alla globalizzazione e, in particolare, dal proliferare di territori sempre più fluidi e complessi, caratteristici del mondo contemporaneo ${ }^{8}$. In effetti, a rimanere centrale, nell'esperienza odierna, è uno dei più evidenti processi legati alla globalizzazione che rinvia alle implicazioni simboliche e materiali delle "frontiere" che, divenute estremamente mobili e complesse, per effetto del loro scomporsi e ricomporsi continuamente, ne generano altre, mettendo in tensione le nozioni d'interno e d'esterno e il significato tradizionale ad esse attribuito (come dimostrano, per esempio, gli studi di Etienne Balibar ${ }^{9}$, Emanuela Fornari ${ }^{10}$, Sandro Mezzadra e Brett Neilson ${ }^{11}$ ).

La storica denominazione Nord e Sud è divenuta un'imprescindibile materia di studio per la geocritica, che ha problematizzato la questione delle frontiere inserendo lo spazio geografico in un processo dialettico, in cui i rapporti sociali definiscono i rapporti economici e in cui, di conseguenza, i rapporti di dominazione hanno un ruolo centrale: da qui emerge e trova giustificazione una definizione plurale della nozione di Sud ${ }^{12}$.

In Italia, le conseguenze e gli effetti della globalizzazione, della mobilità delle frontiere e i cambiamenti di prospettiva Nord-Sud sono evidenti nella produzione della letteratura meridionale che Jean-Noël Schifano definiva, a giusto titolo, come letteratura prodotta da autori culturalmente legati al Sud che scrivono sul Sud: "Che vi siano nati come Elio Vittorini, o che vi abbiano semplicemente vissuto come Carlo Levi, gli scrittori del Sud sono quelli la cui opera si nutre della realtà, dei sogni e dei miti dell'Italia meridionale"13.

Ieri come oggi, la rappresentazione del Sud nella letteratura italiana solleva importanti questioni identitarie legate a complesse nozioni di frontiera, di

8. Cfr. Bauman, Zygmunt, Modernità liquida, Roma-Bari, Laterza, 2007 [2000].

9. Cfr. Balibar, Etienne, Noi cittadini d'Europa? Le frontiere, lo stato, il popolo, a cura di B. Foglio, A. Simone, Roma, Manifestolibri, 2004.

10. Fornari, Emanuela, Linee di confine. Filosofia e postcolonialismo, Torino, Bollati Boringhieri, 2011.

11. Mezzadra, Sandro, Neilson, Brett, Confini e frontiere. La moltiplicazione del lavoro nel mondo globale, Bologna, Il Mulino, 2014.

12. Cfr. Harvey, David, Spaces of Global Capitalism. Towards a Theory of Uneven Geographical Development, New York, Routledge, 2001.

13. "Qu'ils y soient nés comme Elio Vittorini, ou bien qu'ils y aient simplement vécu comme Carlo Levi, les écrivains du Sud sont ceux dont l'œuvre se nourrit de la réalité, des rêves et des mythes de l'Italie méridionale" (Schifano, Jean-Noël, Littérature italienne méridionale», in "Italie. Langue et littérature », in Encyclopédie Universalis France [http://www.universalis-edu.com/]). 
sovranità, di identità, di cittadinanza che si iscrivono in un'idea di Sud sempre più ampia e pluralizzata. In questo volume, tale contesto è ben tradotto da quei saggi che, trattando di letteratura e forme d'arte, propongono perlopiù lo studio di autori sia emblematicamente antesignani di un discorso politico-culturale relativo alle rappresentazioni dei sud, dei suoi confini e delle sue continue dislocazioni identitarie (Carlo Levi) sia promotori di nuovi paradigmi concettuali, etici, tematici e linguistici (Giulio Angioni, Raffaele Puddu, Valeria Parrella, Massimo Maugeri, Livio Romano, Alessandro Leogrande, Maria Marcone, Antonio Campobassso, Nicola Lagioia, Francesco Laudadio, ecc.). Le loro opere, infatti, seppure con diverse modalità, diventano una barriera resistenziale tanto a visioni culturali omologanti quanto a regionalismi monolitici e passatisti o a centralismi politici e identitari fagocitanti, aprendo, il più delle volte, delle linee di fuga verso altri Sud. Poco importa se questo accade attraverso una distorsione del reale operata a livello simbolico o se tale fenomeno si produce tramite un referenziale "ritorno alla realtà".

Quello che importa, invece, sono le nuove fluidità politico-culturali che alimentano le diverse linee finzionali presentate in questo volume, refrattarie a visioni rigidamente circoscritte, al definitivo, all'assoluto e a ogni statica catalogazione. Alcune delle più importanti peculiarità di queste scritture creative, che raccontano il Sud dal Sud, emergono dalle analisi critiche qui proposte che ne sottolineano le raffinate rielaborazioni della memoria proiettate su rizomatiche aggregazioni sovranazionali, le nuove ipotesi comunitarie impostate sulla ricchezza delle differenze culturali o, ancora, le suggestive e variegate cifre espressive e stilistiche che attingono alle fonti di un patrimonio linguistico e culturale plurale. Queste scritture aprono così a una nuova e pragmatica visione del Sud, in grado di superare il problema del meridionalismo nel segno dell'“apprendere" cassaniano, ossia del saper "cambiare destinazione accettando il rischio di un percorso tortuoso"14. La buona strada dell'“apprendere" è stata quindi imboccata da un'arte consapevole e federatrice che, come direbbe ancora Franco Cassano, ci fa "tornare a sperare".

Margherita MARRAS, Université d'Avignon

(CRIX-EA 369 Études Romanes, université Paris Nanterre)

Giuliana PIAs, Université Paris Nanterre (CRIX - EA 369 Études Romanes)

14. Cassano, Franco, "En Attendant Méditérranée”, cit., p. 24. 